\title{
Graphene oxide for biomedical applications
}

\begin{abstract}
Graphene oxide (GO) is one of the most promising functional materials used in various applications like energy storage (batteries and supercapacitors) sensors, photocatalysis, electronics and in biomedicine. The last 10 years literature on GO for biomedical applications revealed and confirmed the scope of its potential capabilities as biomaterial. GO alone and its modified form with different materials (surface functionalization, immobilization of nanoparticles and composite formation) also proved as a multifunctional candidate for medical biotechnology. A material for its use in biomedical applications must be biocompatible and nontoxic to the living cells.. Although there are some concerns about the toxicity of the GO in specific cases, a dosage range and size effects reported in the literature to use it as a nontoxic materials. In view of all these points, an effort has been made to review and emphasize the scope of GO as a biomedical agent for the applications like targeted drug delivery, cancer theranostics, bioimaging and biosensors etc. Further potential applications along with the future scope and limitations of GO have also been highlighted in this review.
\end{abstract}

Keywords: Graphene oxide, Nanocomposites, Toxicity, Biosensors, Biomedicine, Cancer, Drug delivery
Volume 5 Issue 6 - 2017

\section{Santhosh Kumar K, Monami Das Modak,} Pradip Paik

School of Engineering Sciences and Technology, University of Hyderabad, India

Correspondence: Pradip Paik, School of Engineering Sciences and Technology, University of Hyderabad, Telangana, India, Tel 9|-040-23 |3-4457 Email pradip.paik@gmail.com

Received: May 06, 2017 | Published: July 12, 2017

\section{Introduction}

GO is mainly used in biomedicine such as for drug delivery, cancer therapy, imaging and biosensors because of its physico chemical properties and biocompatibiliy. GO possesses unique structure i.e., graphene basal plane is attached with various biocompatible functional groups like carboxylic $(\mathrm{COOH})$ and hydroxyl $(\mathrm{OH})$ etc. The attached functional groups lead to further functionalization and conjugation or immobilization of other nanoparticles on its surface. Further, the size (number of layers, lateral dimension) and shape of GO plays an important role in deciding its properties that can be used in various applications. Thickness gradient of the GO sheets showed various functionality and tunable properties. Based on the reported literature in the past decade, a comprehensive review on the GO's applications in the biomedicine has been summarized in to four main categories, such as

a. Drug delivery and Cancer therapy.

b. Biosensors.

c. Bio-Imaging.

d. Antibacterial activities and the Toxicity effects have also been discussed in the separate section.

\section{Discussion}

\section{Drug delivery and cancer therapy}

Research on GO nanocomposites was reported extensively for its uses in drug delivery and cancer therapy. GO played a significant role in sorting out the drawbacks in dealing with cancer treatment. The extensive loading of anticancer drugs on GO is essentially influenced by the pi-pi stacking interactions. A hybrid nanocomposite of Hypocrellin B (HB) stacked GO can be synthesized through pipi interaction. This nanocomposite generates reactive oxygen species (ROS) efficiently and accelerate the killing of tumor cells under radiation. . Gold (Au) nanorods vesicle@ reduced graphene oxide (rGO) hybrid nanocomposite showed excellent drug release, enhanced photo thermal and photo acoustic effect to treat the cancer cells when loaded with commonly used anticancer drug doxorubicin (DOX).
DOX release can be controlled by the near infrared (NIR) photothermal effect in intracellular acidic environment. This nanocomposite enables efficient inhibition of tumor growth due to sequential drug release and killing of infected cells . ${ }^{2}$

GO on integration with NIR radiation produce heat inside cancer cells. Further it is reported that radionuclide I-131 labeled PEG with $\mathrm{rGO}$ can be used in potential combined therapies, e.g., photo thermal therapy (PTT) and radio therapy to treat the cancer cells. GO on excitation with NIR radiation, induce the photothermal effect while I-131 emits X-rays to kill the cancer cells. Due to this multiple effects the efficient elimination of tumor cells is possible. However, there is a concern on toxicity of this nanocomposites.$^{3}$ A coreshell nanocomposite of chitosan based polyseudorotaxane shell and $\left(\mathrm{Fe}_{3} \mathrm{O}_{4} @ \mathrm{GO} @ \mathrm{mSiO}_{2}\right)$ core showed excellent pH dependent release. The extent of drug release can be controlled by changing the $\mathrm{pH}$ and the bursting of shell at $5.5 \mathrm{pH}$ showed maximum release. Furthermore, this core-shell nanocomposite is quite soluble and formed stable colloid in biological fluids ${ }^{4}$ Hydrophobic drugs can also be delivered to the tumor cells using GO. Water insoluble anticancer drug SN38 (hydrophobic aromatic molecules including a camptothecin (CPT) analogue) can be made soluble for treating cancer cells efficiently by loading them with novel nanocomposite of nano GO with branched polyethylene glycol (NGO@branched PEG). This drug loaded nanocomposite formulation showed high cancer killing potency than that of irinotecan (CPT-11)..$^{5}$

Loading of more amount of drug is possible due to the surface functionality and high surface area of GO. As an example, more amount of drug loading (irinotecan) was possible due to the high surface area of GO in hyaluronic acid/polyaspartamide based double network nanogels@GO nanostructure. This loaded drug can be driven to the target through the changing the $\mathrm{pH}$ of external medium and NIR irradiation and can be treated the human colon cancer . ${ }^{6}$ rGO blending with alginate@chitosan derivatives (rGO@CSD) hydrogel is also a potential candidate for delivery of various small drug molecules. This nanocomposite exhibited a high drug loading efficiency of $\sim 82.8 \%$ for small molecule fluorescein sodium (FL) $\mathrm{CSD} / \mathrm{rGO}$ /alginate and also showed negligible cytotoxicity to hepatic stellate cell lines .? A thermo sensitive nanogel of N-isopropyl acrylamide (NIPAM) 
modified GO nanocomposite showed potential for high drug loading capacity and excellent drug release behaviour at high temperature. It was also observed that there was no burst release with temperature ${ }^{8}$ Magnetically $\left(\mathrm{Fe}_{3} \mathrm{O}_{4}\right)$ modified $\mathrm{GO}$ functionalized with chitosan and methoxy poly (ethylene glycol) mPEG-NHS nanostructure was used for targeted drug delivery of chemotherapy drugs CPT-11 and DOX. High drug loading and $\mathrm{pH}$ dependent drug release properties are promising for targeted drug delivery and cancer therapy.$^{9} \mathrm{GO}$ $\mathrm{Fe}_{3} \mathrm{O}_{4}$ super paramagnetic drug carrier was developed with loading of $18.6 \mathrm{wt} \%$ of DOX hydrochloride. The loading capacity was as high as $1.08 \mathrm{mg} / \mathrm{mg}$. The loaded and unloaded nanocomposites showed good hydrophilicity. Aggregation under acidic conditions can be regulated by applying external magnetic fields.${ }^{10} \mathrm{CpG}$ oligodeoxynucleotides (ODNs) loading capacity was increased on GO-chitosan nanocomposites and showed lower cytotoxity compared with GO. Loading of CpG-ODNs was due to the electrostatic interaction between CpG-ODNs and GO-CS . ${ }^{11}$ Moreover, nano GO (NGO) sheets showed lesser cytotoxicity when they were used for drug delivery and cancer treatment. Folic acid modified NGO loaded with dual drugs DOX and camptothecin (CPT) showed remarkable high toxicity to (Michigan cancer foundation-7) MCF-7 cells, while these drugs are loaded with pristine NGO showed lesser cytotoxicity. The loading ability of this nanostructure for multiple drugs enable us its potential use in biomedicine.$^{12}$

Multifunctional nanocomposites have also been reported based on the GO for drug loading, cancer treatment and imaging etc. [mPEG]@ NGO was prepared for loading of photosensitizer zinc phthalocyanine $(\mathrm{ZnPc})$ towards treating cancer cells MCF-\& through photodynamic therapy (PDT). Though $\mathrm{ZnPc}$ is hydrophobic, it is internalized in MCF-7 cells with the addition of NGO-mPEG nanocomposite.${ }^{13}$ Low molecular weight branched polyethylenimine (BPEI) attached GO showed an improved DNA binding, condensation and transfection efficiency compared to high molecular weight BPEI. Due to the tunability of GO, this BPEI@GO hybrid nanocomposite could be extended to SiRNA delivery and PTT.${ }^{14}$ Light controllable CpGODNs delivery was proposed and showed excellent photothermal and immunological effects towards the cancer cell tumor reduction in GO@PEG and PEI nanocomposites . ${ }^{15}$ Through electrostatic selfassembly of functionalized GO with chitosan and sodium alginate a nanocomposite was formed and loaded with DOX, and showed $\mathrm{pH}$ dependent drug release behavior. Remarkable inhibition of MCF7 cancer cells was also reported using this nanocomposite . ${ }^{16} \mathrm{GO} @$ sodium alginate nanohybrid showed potential for DOX loading and exhibited profound cytotoxicity to HeLa cells. A very high loading capacity of $1.843 \mathrm{mg} / \mathrm{mg}$ was obtained for this nanocomposite under the physiological conditions of $\mathrm{pH} 6.5$ and 7.4.${ }^{17}$ Authors of this work reported that an anticancer effect of 13 times more than the previous study was obtained using $\mathrm{GO} @ \mathrm{SiO}_{2} / \mathrm{TiO}_{2}$ nanocomposite. Multi functionality of this nanocomposite showed potential for loading of anticancer drug protoporphyrinIX and efficient for both PDT and PTT ${ }^{18}$ A nanocomposite of rGO@phosphorescent PEG modified Ru (II) complex (Ru-PEG) also developed and is very effective for both PTT and PDT. This nanocomposite showed high efficacy towards multimodal imaging and treatment of the cancer cells through the generation of ROS.${ }^{19}$ GO-folic acid (FA)/bovine serum albumin (BSA) nanocomposite loaded with DOX showed potential role in treating the cancer cells..$^{20}$

The strategy of core-shell formation was also employed for designing the multifunctional GO nanocomposites for drug delivery and cancer treatment. Core-Shell nanostructure of upconversion nanoparticles (UCNP) and GO quantum dots were synthesized and used for imaging as well as cancer treatment. A photosensitizer was loaded for PDT and a chemotherapy drug (hypocrellin A) was also attached on GO quantum dots and combined with PEGlayted UCNP. This nanostructure was multifunctional for cell imaging, drug delivery and cancer therapy.$^{21}$ A multifunctional nano structure consisting of fluorescein loaded zeolitic imidazolte frameworks-8 with $\mathrm{GO}$ was developed for simultaneous $\mathrm{pH}$ controlled drug release and PTT. This nanostructure showed high efficacy in killing cancer cells when irradiated with 808 nm near NIR.22 GO@chlorogenic acid nanocomposite showed cell viability $>80 \%$ to the normal cells and in contrary showed an enhanced toxicity towards cancer cells (HepG2, A549 and HeLa cells) ${ }^{23}$ rGO has been synthesized by hydrothermal reduction (nontoxic) and surfactant free dispersion was obtained by applying ultrasonication. Stable rGO dispersion was obtained by optimizing the ultrasonic conditions and was used for an efficient delivery of anti-cancer drug Paclitaxel ${ }^{24}$ A composite of nano-sized graphene oxide and gold (NGO@Au) was synthesized and GO was attached with folic acid. This nanocomposite was used for targeting the cancer cells and simultaneous release of loaded DOX and Au nanoparticles which substantially increased the inhibition of cancer cells upon exposure of NIR radiations (in vivo).$^{25}$

\section{Biosensors}

GO based sensors were developed and used for various biological moieties detection. Especially the fluorescence quenching nature of GO played an important role in enhancing the sensitivity of the probe. Further, GO was used to detect mostly DNA with low background signal. GO and silver $(\mathrm{Ag})$ ions nanocomposite was used for the detection of bacteria. The Ag ions diffused from the composite and kill the bacteria. The efficiency of this sensor was tested through a electrochemical method and compared with conventional Dot blot assay . ${ }^{26}$ A nanocomposite of GO@Au nanorods was synthesized to detect the DNA. DNA sensor based on this nanocomposite showed high selectivity and distinguished complementary DNA sequences from huge amount of single-base mismatched DNA (1000:1).$^{27}$ DNA templated click chemistry strategy based on GO was proposed for the fluorescence detection of $\mathrm{Cu} 2+$. Low detection limit was obtained under the optimal conditions. The specificity of the click chemistry and GO quenching ability revealed low detection limit.$^{28}$

A hydrogel of GO@fish sperm DNA was used for mitochondrial (label-free) DNA detection by measuring the conductivity through the impedance analysis. The conductivity of this hydrogel can be tuned by varying the GO's properties. Moreover, this hydrogel electrode showed potential for the deduction of ovarian cancer cells DNA samples . ${ }^{29}$ DNA immobiliized on GO glassy electrode nanocomposite was proposed for the detection of acrylamide (AA). Due to the excellent electron transfer ability of GO, sensing ability of this nanocomposite was linear to the concentration of DNA.$^{30}$ Double strand DNA was directly detected without denaturation by using peptide nucleic acid (PNA) modified graphene oxide as a fluorescence quencher. This composite showed very low background signal, sequence selectivity, high sensitivity and tighter turn-on signal control.$^{31}$ There are some reports even on adenosine triphosphate (ATP) sensing by GO based nanocomposites. An integrated GO and hairpin shaped molecular aptamer beacon (MAB) nanocomposite was proposed and showed quenched fluorescence intensity in the absence of ATP. When this nanocomposite is interacted with ATP, quenched fluorescence intensity was recovered and low background signal was observed ${ }^{32}$ Rather than physisorbed aptamer probes, covalently linked aptamer probes (fluorophore and amino dual modified) showed better detection of ATP. Covalently linked aptamer probes on GO showed higher fluorescence signal when imaging intracellular ATP.$^{33}$ 


\section{Bio-imaging}

GO was proved to be a potential bioimaging tool for tumor cells Tuning the lateral size of the GO in the nano range yields fluorescent property to it. A luminescent single layered NGO was developed and showed promising applications as live cell imaging agent in the NIR and visible range with low background signal. Moreover pi-pi stacking of anti-cancer drug (e.g., DOX) was found suitable for killing cancer cells in vitro.34 A nanocomposite GO@ aptamercarboxyfluorescein (FAM) was fascinated for probing in living cells and this nanocomposite showed great sensing, protecting capabilities in living cells.$^{35}$

\section{Antibacterial activity}

GO composites are also promising candidates for antibacterial activities. Few GO-based nanocomposites evidenced better antibacterial activities compared to the existing materials. chitosan@ AgNPs@GO nanocomposite exhibited highly efficient antibacterial activity towards the bacteria (methicillin-resistant Staphylococcus aureus) strains. This nanocomposite showed better antibacterial activity than the AgNPs or GO@AgNP materials . ${ }^{36}$ GO- zinc oxide $(\mathrm{ZnO})$ nanofillers filled poly lactic acid (PLA) matrix nanocomposite showed multiple improved properties like mechanical strength and antibacterial activity.$^{37}$

\section{Toxicity}

Toxicity and biocompatibility are the prior concerns for the biomedical materials. There were various studies on the toxicity and biocompatibility of the GOs and its derivatives. Toxicity and in vivo biodistribution of NGO@poly sodium 4-styrenesulfonate (PSS) nanocomposite was studied for 6 months after intravenously injecting in mice. Potential accumulation of NGO@PSS was observed in the lung, liver and spleen. Upon accumulation, injury and chronic inflammation of these parts confirms the toxicity of this nanocomposite.$^{38}$ The effect of size and concentration of GO sheets on the cytotoxicity of normal cells and cancer cell were elucidated. Especially low concentrations of the GO showed negligible toxicity.

rGO was synthesized by sonication and followed by reduction of PEGlayted GO sheets. Micron and nano sized (lateral) rGO sheets were obtained and tested for cyto and geno toxic effects. A high concentration of $100 \mathrm{mg} / \mathrm{mL}$ after $1 \mathrm{~h}$ exposure time, cytotoxic effect was observed and this study showed the effect of concentration of rGO cytotoxicity.$^{39}$ Toxicity of bare GO was evaluated in male rats and observed after seven days of injection with different concentrations of GO which causes inflammation in lung, spleen and liver. This study proposed that low concentration of GO is nontoxic.$^{40}$ A lower cytotoxicity was observed in the graphene synthesized from reduction of GO using pectin from Tithonia divesifolia.$^{41} \mathrm{GO}$ was synthesized by unzipping the single wall carbon nanotubes (CNT) and cytotoxicity was examined towards human neuroblastoma cells. The cytotoxicity of this GO was checked with SK-N-BE (2) and SH-SY5Y cell lines and found low concentration of GO gives minimal effects on healthy cells.$^{42} \mathrm{GO}$ can be single layer and multilayer and the number of layers definitely changes its physico chemical properties and the studies were done in this regard also. Cytotoxicity of single layered GO (SLGO) and multi layered GO (MLGO) was investigated systematically. The dependence of size and dose of SLGO and MLGO towards toxicity was studied in the presence /absence of pluoronic F-127 on THP-1 cells. This study revealed that prior consideration of GO concentration and dose need to be optimized preceding to biomedical applications.$^{43}$ To counter the effects of GO toxicity, it has been further functionalized and showed no toxicity. A nontoxic and biocompatible nanostructure of PEGlayted rGO was developed and studied with mouse bone marrow stem cells. No increase in ROS found and confirmed by cell function ${ }^{44}$ From the above information, it is clear that GO is a potential candidate for biomedical applications and eventually low concentration of GO is nontoxic.

Other than the above mentioned potential applications, GO also showed mutagenesis, scaffold generation, anti-tuberculosis, aromatic hydrocarbons extractions from food samples, glucose sensitivity and triggered growth in plant when treated with it. In vivo, in vitro studies of GO injected intravenously in mice showed mutagenesis compared to the cyclophosphamide, a classic mutagen. This study reveals an extra control needs to be taken on the doses of the GO injection.$^{45}$ Five times enhanced growth was observed with $20 \mathrm{mg} / \mathrm{L}$ of GO while untreated tobacco roots showed lesser growth. Treating tobacco seedlings with GO affected the gene transcript levels of the IAAA relatives allowed enhanced root growth. ${ }^{46} \mathrm{GO} @ \mathrm{BSA}$ showed strong binding with vascular endothelial growth factor (VEGF) resulting in blocking the interaction between VEGF and their receptors which further results in reduced/blocked blood vessel formation in rabbit. So from this, GO can act as anti-angiogenic agent ${ }^{47} \mathrm{~A}$ highly hydrophilic nanostructure of $\mathrm{COOH}$ groups functionalized $\mathrm{GO}$ with beta-cyclodextrin was proposed and showed dispersion stability for 12 months, which will make them applicable for biology and medicine . ${ }^{48}$ Graphene derived from removal of oxygen functional groups of GO showed potential as anti-tuberculosis agent towards M. Tuberculosis (M. TB) H37Ra ${ }^{49}$ Wound healing nanocomposite fibers based on GO with poly(2-hydroxyethyl methacrylate)-graft-poly(epsilon-caprolactone) [P(HEMA-g-CL)] was proposed and showed in vitro degradability, hydrophilicity, conductivity and biocompatibility towards application as nano scaffolds for regenerative medicine.$^{50}$ Peptide (arginineglycine-aspartic acid) modified GO showed better biocompatibility than the pristine GO. At the same time, AMP (Antimicrobial peptide modified Go showed potential for sterilization.$^{51} 16$ polycyclic aromatic hydrocarbons (PAHs) were extracted from vegetable oil by using nanocomposite of 3D ionic liquid functionalized magnetic GO. Compared to existing molecularly imprinted solid phase extraction method (MSPE), MSPE based on this nanocomposite is cost effective and efficient for light PAHs extraction.$^{52}$ GO-BSA nanocomposite hydrogel showed better $\mathrm{pH}$ glucose sensitivity and lower initial burst release compared to HTCC/BSA and HTCC/2.0wMGOBSA hydrogels.$^{53}$ PEGlayted GO-hemin composite showed higher peroxidase like activity than bare PEG@GO composite. ${ }^{54}$

\section{Conclusion}

GO's potential applications in the field of biomedicine have been revealed based on the reported works in the past few years. GO's structure, size and shape played an important role in various applications. Because of the high surface area a large number of nanoparticles or biomolecules were immobilized on it that together lead to multifunctional nanocomposites. The size reduction of GO yielded it to be a fluorescence bio marker. The various functional groups attached to GO increase its dispersity in various bio fluids. Additionally, hydrophobic drug loaded GO can easily be delivered into the cancer cells for enhancing the efficiency of treatment. Though there are some concerns about GO's cytotoxicity, the tunability of the size and dose made this material nontoxic and biocompatible. These findings in the literature enable one to recognize and use GO's tremendous applicability in biomedicine. However, there is still a lot of scope to elevate its potential. Studies based on the GO functionalized with single type of oxygen functional group for different cancer cells should be focused and it can be further checked with other oxygen moiety to the different cancer cells. 


\section{Conflicts of interest}

None.

\section{Acknowledgments}

None.

\section{References}

1. Zhou L, Jiang H, Wei S, Ge X, Zhou J, et al. High-efficiency loading of hypocrellin B on graphene oxide for photodynamic therapy. Carbon. 2012;50(15):5594-5604.

2. Song J, Yang $X$, Jacobson $O$ et al. Sequential Drug Release and Enhanced Photothermal and Photoacoustic Effect of Hybrid Reduced Graphene Oxide-Loaded Ultrasmall Gold Nanorod Vesicles for Cancer Therapy. ACS Nano. 2015;9(9):9199-9209.

3. Chen L, Zhong X, Yi X et al. Radionuclide I-131 labeled reduced graphene oxide for nuclear imaging guided combined radio- and photothermal therapy of cancer. Biomaterials. 2015;66:21-28.

4. Pourjavadi A, Tehrani ZM, Jokar S Chitosan based supramolecular polypseudorotaxane as a $\mathrm{pH}$-responsive polymer and their hybridization with mesoporous silica-coated magnetic graphene oxide for triggered anticancer drug delivery. Polymer (Guildf). 201;576:52-61.

5. Liu Z, Robinson J T, Sun X, Dai H PEGylated nanographene oxide for delivery of water-insoluble cancer drugs. $J$ Am Chem Soc. 2008;130(33):10876.

6. Fiorica C, Mauro N, Pitarresi G et al. Double-Network-Structured Graphene Oxide-Containing Nanogels as Photothermal Agents for the Treatment of Colorectal Cancer. Biomacromolecules. 2017;18(3):1010-1018.

7. Chen $\mathrm{K}$, Ling $\mathrm{Y}$, Cao $\mathrm{C}$ et al.Chitosan derivatives/reduced graphene oxide/alginate beads for small-molecule drug delivery. Mater Sci Eng C-Materials Biol Appl. 2016;69:1222-1228.

8. Bardajee GR, Hooshyar Z Drug release study by a novel thermo sensitive nanogel based on salep modified graphene oxide. J Polym Res. 2017;24(3):1.

9. Huang YS, Lu YJ, Chen J-P Magnetic graphene oxide as a carrier for targeted delivery of chemotherapy drugs in cancer therapy. J Magn Magn Mater. 2017;427:34-40.

10. Yang X, Zhang X, Ma Y et al. Superparamagnetic graphene oxide$\mathrm{Fe}_{3} \mathrm{O}_{4}$ nanoparticles hybrid for controlled targeted drug carriers. J Mater Chem. 2009;19(18):2710-2714.

11. Zhang H, Yan T, Xu S et al. Graphene oxide-chitosan nanocomposites for intracellular delivery of immunostimulatory $\mathrm{CpG}$ oligodeoxynucleotides. Mater Sci Eng C-Materials Biol Appl. 2017;73:144-151.

12. Zhang L, Xia J, Zhao Q, Liu L, Zhang Z Functional graphene oxide as a nanocarrier for controlled loading and targeted delivery of mixed anticancer drugs. Small. 2010;6(4):537-544.

13. HaiQing D, ZhiLei Z, HuiYun W et al. Poly (ethylene glycol) conjugated nano-graphene oxide for photodynamic therapy. Sci China-Chemistry. 2010;53(11):2265-2271.

14. Kim H, Namgung R, Singha K et al. Graphene Oxide-Polyethylenimine Nanoconstruct as a Gene Delivery Vector and Bioimaging Tool. Bioconjug Chem. 2011;22(12):2558-2567.

15. Tao Y, Ju E, Ren J, Qu X Immunostimulatory oligonucleotidesloaded cationic graphene oxide with photothermally enhanced immunogenicity for photothermal/immune cancer therapy. Biomaterials 2014;35(37):9963-9971.

16. Lei $\mathrm{H}$, Xie M, Zhao $\mathrm{Y}$, Zhang $\mathrm{F}$ et al. Chitosan/sodium alginate modificated graphene oxide-based nanocomposite as a carrier for drug delivery. Ceram Int. 2016;42(150:17798-17805.
17. Fan L, Ge H, Zou S, Xiao Y, Wen H, et al. Sodium alginate conjugated graphene oxide as a new carrier for drug delivery system. Int $\mathrm{J}$ Biol Macromol 2016;93(PT A):582-590.

18. Jang Y, Kim S, Lee Set al. Graphene Oxide Wrapped $\mathrm{SiO}_{2} / \mathrm{TiO} 2$ Hollow Nanoparticles Loaded with Photosensitizer for Photothermal and Photodynamic Combination Therapy. Chem Eur J 2017;23:3719-3727.

19. Zhang DY, Zheng Y, Tan CP et al. Graphene Oxide Decorated with $\mathrm{Ru}(\mathrm{II})-$ Polyethylene Glycol Complex for Lysosome-Targeted Imaging and Photodynamic/Photothermal Therapy. ACS Appl Mater Interfaces. 2017;9(8):6761-6771.

20. Ma N, Liu J, He W et al. Folic acid-grafted bovine serum albumin decorated graphene oxide: An efficient drug carrier for targeted cancer therapy. J Colloid Interface Sci. 2017;490:598-607.

21. Choi SY, Baek SH, Chang SJ et al. Synthesis of upconversion nanoparticles conjugated with graphene oxide quantum dots and their use against cancer cell imaging and photodynamic therapy. Biosens Bioelectron. 2017;93:267-273.

22. Tian Z, Yao X, Zhu Y Simple synthesis of multifunctional zeolitic imidazolate frameworks-8/graphene oxide nanocrystals with controlled drug release and photothermal effect. Microporous Mesoporous Mater. 2017;237:160-167.

23. Barahuie F, Saifullah B, Dorniani D et al. Graphene oxide as a nanocarrier for controlled release and targeted delivery of an anticancer active agent, chlorogenic acid. Mater Sci Eng C-materBiol Appl. 2017;74:177-185.

24. Bai RG, Muthoosamy K, Shipton FN et al. Acoustic cavitation induced generation of stabilizer-free, extremely stable reduced graphene oxide nanodispersion for efficient delivery of paclitaxel in cancer cells. Ultrason Sonochem. 2017;36:129-138.

25. Chauhan G, Chopra V, Tyagi A et al. Gold nanoparticles compositefolic acid conjugated graphene oxide nanohybrids' for targeted chemothermal cancer ablation: In vitro screening and in vivo studies. Eur $J$ Pharm Sci. 2017;96:351-361.

26. Wan Y, Wang Y, Wu J, Zhag D Graphene Oxide Sheet-Mediated Silver Enhancement for Application to Electrochemical Biosensors. Anal Chem. 2011;83(3):648-653.

27. Han X, Fang X, Shi A et al. An electrochemical DNA biosensor based on gold nanorods decorated graphene oxide sheets for sensing platform. Anal Biochem. 2013;443(2):117-123.

28. Zhou L, Shen Q, Zhao P et al. Fluorescent detection of copper(II) based on DNA-templated click chemistry and graphene oxide. Methods. 2013;64(3):299-304.

29. Sun L, Hu N, Peng J et al. Ultrasensitive Detection of Mitochondrial DNA Mutation by Graphene Oxide/DNA Hydrogel Electrode. $A d v$ Funct Mater. 2014;24:6905-6913.

30. Li D, Xu Y, Zhang L, Tong H A Label-free Electrochemical Biosensor for Acrylamide Based on DNA Immobilized on Graphene Oxide-Modified Glassy Carbon Electrode. Int J Electrochem Sci. 2014;9:7217-7227.

31. Lee J, Park IS, Jung E, Lee Y, Min D-H Direct, sequence-specific detection of dsDNA based on peptide nucleic acid and graphene oxide without requiring denaturation. Biosens Bioelectron. 2014;62:140-144.

32. He Y, Wang ZG, Tang HW et al. Low background signal platform for the detection of ATP: When a molecular aptamer beacon meets graphene oxide. Biosens Bioelectron. 2011;29(1):76-81.

33. Liu Z, Chen S, Liu B et al. Intracellular Detection ofATPUsing an Aptamer Beacon Covalently Linked to Graphene Oxide Resisting Nonspecific Probe Displacement. Anal Chem. 2014;86(24):12229-12235.

34. Sun X, Liu Z, Welsher K et al. Nano-Graphene Oxide for Cellular Imaging and Drug Delivery. Nano Res 2008;1(3):203-212. 
35. Wang Y, Li Z, Hu D, Lin CT, Li J, et al. Aptamer/Graphene Oxide Nanocomplex for in Situ Molecular Probing in Living Cells. J Am Chem Soc. 2010;132(27):9274-9276.

36. Marta B, Potara M, Iliut M et al. Designing chitosan-silver nanoparticlesgraphene oxide nanohybrids with enhanced antibacterial activity against Staphylococcus aureus. Colloids Surfaces A-Physicochemical Eng Asp. 2015;487:113-120.

37. Huang Y, Wang T, Zhao X et al. Poly (lactic acid)/graphene oxide$\mathrm{ZnO}$ nanocomposite films with good mechanical, dynamic mechanical, anti-UV and antibacterial properties. J Chem Technol Biotechnol. 2015;90(9):1677-1684.

38. Wen KP, Chen YC, Chuang $\mathrm{CH}$ et al. Accumulation and toxicity of intravenously-injected functionalized graphene oxide in mice. $J \mathrm{Appl}$ Toxicol. 2015;35(10):1211-1218.

39. Akhavan O, Ghaderi E, Akhavan A Size-dependent genotoxicity of graphene nanoplatelets in human stem cells. Biomaterials. 2012;33(32):8017-8025.

40. Li Y, Wang Y, Tu L et al. Sub-Acute Toxicity Study of Graphene Oxide in the Sprague-Dawley Rat. Int J Environ Res Public Health. 2016;13(11):E1149.

41. Tran TTT, Le HNT, Tran H Van et al. Tithonia diversifolia pectin - reduced graphene oxide and its cytotoxic activity. Mater Lett. 2016;183:127-130.

42. Mari E, Mardente S, Morgante E et al. Graphene Oxide Nanoribbons Induce Autophagic Vacuoles in Neuroblastoma Cell Lines. Int J Mol Sci. 2016;17(12):E1995.

43. Cho Y C, Pak P J, Joo Y H et al. In vitro and in vivo comparison of the immunotoxicity of single- and multi-layered graphene oxides with or without pluronic F-127. Sci Rep. 2016;6:38884.

44. Syama S, Aby CP, Maekawa T, et al. Nano-bio compatibility of PEGylated reduced graphene oxide on mesenchymal stem cells. IOP Scinece. 2017;4(2):1
45. Liu Y, Luo Y, Wu J et al. Graphene oxide can induce in vitro and in vivo mutagenesis. Sci Rep. 2013;3:3469.

46. Jiao J, Yuan C, Wang J et al. The Role of Graphene Oxide on Tobacco Root Growth and Its Preliminary Mechanism. J Nanosci Nanotechnol. 2016;16:12449-12454.

47. Lai PX, Chen CW, Wei SC et al. Ultrastrong trapping of VEGF by graphene oxide: Anti-angiogenesis application. Biomaterials. 2016;109:12-22.

48. Meng N, Su Y, Zhou N et al. Carboxylated graphene oxide functionalized with beta-cyclodextrin-Engineering of a novel nanohybrid drug carrier. Int J Biol Macromol. 2016;93(Pt A):117-122.

49. Han W, Niu WY, Sun B et al. Biofabrication of polyphenols stabilized reduced graphene oxide and its anti-tuberculosis activity. $J$ Photo chem Photobiol B-Biology. 2016;165:305-309.

50. Massoumi B, Ghandomi F, Abbasian M et al. Surface functionalization of graphene oxide with poly(2-hydroxyethyl methacrylate)-graftpoly(epsilon-caprolactone) and its electrospun nanofibers with gelatin. Appl Phys A-Materials Sci Process. 2016;122(12):13.

51. Shi L, Wang L, Chen J et al. Modifying graphene oxide with short peptide via click chemistry for biomedical applications. Appl Mater Today. 2016;5:111-117.

52. Zhang Y, Zhou H, Zhang ZH et al. Three dimensional ionic liquid functionalized magnetic graphene oxide nanocomposite for the magnetic dispersive solid phase extraction of 16 polycyclic aromatic hydrocarbons in vegetable oils. J Chromatogr A. 2017;1489:29-38.

53. Zhao X, Zou X, Ye L Controlled $\mathrm{pH}$ and glucose-responsive drug release behavior of cationic chitosan based nano-composite hydrogels by using graphene oxide as drug nanocarrier. J Ind Eng Chem. 2017;49:36-45.

54. Zhang W, Sun Y, Lou Z et al. In vitro cytotoxicity evaluation of graphene oxide from the peroxidase-like activity perspective. Colloids and Surfaces B-Biointerfaces. 2017;151:215-223. 